\title{
Funcionamento 24 horas para Unidades de Saúde da Família: uma solução para ampliação de acesso? Um ensaio sobre as "Upinhas" do Recife
}

\author{
24-hour operation for Family Health Units: a solution for access expansion? \\ An essay on the "Upinhas" of Recife \\ Funcionamiento 24 horas para las Unidades de Salud de la Familia: ¿Una solución para la \\ ampliación del acceso? \\ Un ensayo sobre las "Upinhas" de Recife
}

Bruno Henrique Soares Pessoa ${ }^{a}$, Eneline de Andrade Heráclio Gouveiaa ${ }^{a}$ Isabel Brandão Correia ${ }^{a}$

\section{Resumo}

A Atenção Primária à Saúde é via preferencial de acesso ao sistema de saúde, tendo em vista seu papel ordenador, os benefícios da continuidade do cuidado e resolutividade deste nível de atenção. Diversas barreiras de acesso, dentre eles o horário restrito de funcionamento, fazem com que esse primeiro contato não seja facilitado. No Recife, novos equipamentos de Atenção Primária à Saúde vêm sendo implantados desde o ano de 2013: as "Upinhas 24 horas". O objetivo deste trabalho é analisar o modelo "Upinha" apresentado como uma solução para ampliação de acesso. Foi realizada uma revisão da literatura sobre diversas estratégias existentes de ampliação do acesso, a partir das quais se analisou criticamente o modelo "Upinha 24 horas" às lentes dos modelos vigentes. Horário estendido, acesso avançado e acolhimento à demanda espontânea foram os modelos de ampliação de acesso revisados para embasar a discussão. O impacto do horário estendido na ampliação do acesso pode ser minimizado se outras medidas, como mudanças no modelo de agendamento, priorizando o acesso avançado, não forem concomitantemente implantadas. O modelo "Upinha 24 horas", da forma como se apresenta, parece ser baseado na concepção de uma Atenção Primária à Saúde que funciona como complemento para "desafogar" os serviços de atendimento às urgências já existentes e não como ordenadora do serviço de atenção às urgências. Apesar de apontar numa direção inicialmente acertada, a implantação das "Upinhas 24 horas" parece ainda ser uma proposta incipiente na garantia de acesso.

\section{Abstract}

Primary Health Care is a preferential route of access to the health system, considering its ordering role, the benefits of continuity of care and the effectiveness of this level of care. Several access barriers, including restricted hours of operation, make this first contact difficult. In Recife, new equipment for Primary Health Care has been implemented since 2013: the "Upinhas 24 horas". The objective of this essay is to analyze the "Upinha" model presented as a solution to increase access. A review of the literature on several existing strategies of access expansion was carried out, from which the "Upinha 24 horas" model was analyzed critically through the lenses of the current models. Extended hours, advanced access and welcoming of the spontaneous demand were the models of access expansion revised to support the discussion. The impact of extended hours on increased access can be minimized if other measures, such as changes to the scheduling model prioritizing advanced access, are not implemented concurrently. The "Upinha 24 horas" model, as presented, seems to be based on the conception of a Primary Health Care system that works as a complement to "unload" the services of attendance to emergency services and not as ordering of the system emergency attention. Despite pointing in an initially correct direction, the implementation of the "Upinhas 24 horas" still seems to be an incipient proposal in the guarantee of access.

Como citar: Pessoa BHS, Gouveia EAH, Correia IB. Funcionamento 24 horas para Unidades de Saúde da Família: uma solução para ampliação de acesso? Um ensaio sobre as "Upinhas" do Recife. Rev Bras Med Fam Comunidade. 2017;12(39):1-9. http://dx.doi.org/10.5712/rbmfc12(39)1529
Palavras-chave:

Plantão Médico

Controle de Acesso

Atenção Primária à Saúde

Medicina de Família

e Comunidade

Keywords:

After-Hours Care

Gatekeeping

Primary Health Care

Family Practice

Fonte de financiamento: declaram não haver. Parecer CEP: não se aplica. Conflito de interesses: declaram não haver. Procedência e revisão por pares: revisado por pares. Recebido em: 09/05/2017 Aprovado em: 30/08/2017. 


\section{Resumen}

La Atención Primaria es la vía preferencial de acceso al sistema de salud, teniendo en cuenta su papel ordenador, los beneficios de la continuidad del cuidado y resolución de este nivel de atención. Diversas barreras en el acceso, dentro de ellas, el horario restringido de funcionamiento, hacen que ese primer contacto sea difícil. En Recife, nuevos equipos de Atención Primaria vienen siendo implementados desde el año 2013: las "Upinhas 24 horas". El objetivo de este trabajo es analizar el modelo "Upinha" presentado como una solución que amplía el acceso. Fue realizada una revisión de la literatura sobre diversas estrategias existentes de ampliación del acceso, a partir de las cuales se analizó críticamente el modelo "Upinha 24 horas" a la vista de los modelos vigentes. Horario extendido, acceso avanzado y acogimiento de la demanda espontanea, fueron los modelos de ampliación del acceso revisados para sustentar la discusión. El impacto del horario extendido en cuanto a la ampliación del acceso puede ser minimizado si otras medidas, como cambios en el modelo de turnos priorizando el acceso avanzado, no fueran concomitantemente implementadas. El modelo "Upinha 24 horas" de la forma que se presenta, parece basado en la concepción de una Atención Primaria que funciona como complemento para "desahogar" los servicios de atención de urgencias. A pesar de apuntar en una dirección inicialmente correcta, la implementación de las "Upinhas 24 horas" parece ser todavía una propuesta incipiente en la garantía del acceso.
Palabras clave:

Atención Posterior

Control de Acceso

Atención Primaria de Salud Medicina Familiar

y Comunitaria

\section{Introdução}

O acesso de primeiro contato, funcionando como porta de entrada para o sistema de saúde, é um atributo essencial da Atenção Primária à Saúde (APS). ${ }^{1}$ No Brasil, o acesso ao sistema de saúde pode se dar de várias maneiras: via Unidades de Saúde da Família (USF) e Unidades Básicas Tradicionais (UBT), que compõem a APS; via serviços de emergência, tais quais as Unidades de Pronto Atendimento (UPA) e os setores de emergência dos hospitais; e pelo acesso direto ao especialista, este último mais comumente encontrado na saúde suplementar. ${ }^{2}$

AAPS é a via preferencial de acesso ao sistema de saúde, tendo em vista seu papel ordenador, os benefícios da continuidade do cuidado e resolutividade deste nível de atenção ${ }^{3}$ e o excesso de utilização de serviços de emergência por problemas não urgentes. ${ }^{4}$ No entanto, existe uma percepção de que o acesso às unidades de APS não se dá de forma tão facilitada quanto deveria. Essa dificuldade é maior para aqueles que trabalham em horário convencional, havendo um choque de seu horário de trabalho com o horário de funcionamento desses serviços. Cerca de $65 \%$ de todas as consultas em departamento de urgência hospitalar ocorrem entre as 17 horas e as 8 horas da manhã e aos finais de semana, ${ }^{5}$ horários em que habitualmente as unidades de APS não estão em funcionamento.

A dificuldade não se deve apenas ao horário restrito de funcionamento das USF. Uma outra barreira de acesso à população na APS se dá pelo processo de trabalho das equipes, especialmente nos modelos de agendamento. As dificuldades de agendamento de consulta médica fazem com que exista uma lacuna entre a demanda por atendimento e o momento da oferta deste serviço. Quanto mais esse cuidado for adiado, maior será a ameaça à sua qualidade. ${ }^{6}$

Na cidade do Recife, novas USF vêm sendo implantadas desde o ano de 2013: as "Upinhas 24 horas". Esses equipamentos possuem horário de atendimento estendido para as consultas, das $7 \mathrm{~h}$ às $19 \mathrm{~h}$, e 24 horas para as pequenas urgências. ${ }^{7} \mathrm{O}$ objetivo deste trabalho é analisar o modelo "Upinha" exibido como uma solução para ampliação de acesso na Rede de Saúde do Recife. Inicialmente, será apresentada uma revisão da literatura sobre diversas estratégias existentes de ampliação do acesso, para que então se analise criticamente o modelo "Upinha 24 horas" às lentes dos modelos vigentes. 


\section{Ampliando o acesso}

Várias estratégias vêm sendo pensadas para ampliar o acesso no âmbito da APS, visando torná-lo o mais fácil e próximo possível do momento da demanda do usuário. Dentre essas, destacam-se o acesso avançado, o acolhimento à demanda espontânea e o horário estendido.

\section{Acesso avançado}

Preocupados com a lacuna, em média 55 dias, existente entre a demanda por agendamento e o momento da oferta de uma vaga, o departamento de APS de um consórcio de saúde da Califórnia desenvolveu um sistema de agendamento conhecido por acesso avançado, também denominado "acesso aberto" ou "agendamento para o mesmo dia". O sistema consiste em oferecer o atendimento às pessoas no mesmo dia em que elas procuram o serviço, ou em até 24 horas. ${ }^{6}$

Os possíveis benefícios do acesso avançado, para além da redução do tempo de espera por uma consulta, abrangeriam promover a continuidade do cuidado, diminuir as taxas de absenteísmo, aumentar a satisfação de profissionais e usuários, entre outros. No entanto, são atribuídos a esse tipo de sistema de acesso características tais quais a perda da qualidade do seguimento de pacientes com doenças crônicas, a difícil implantação e uma demanda que supera a oferta por serviços de saúde. ${ }^{8}$

Uma revisão sistemática de 24 estudos avaliou os resultados da implementação deste sistema de acesso. ${ }^{8} \mathrm{O}$ tempo de espera para a próxima terceira vaga disponível foi o desfecho que mais consistentemente se reduziu. Houve pequeno benefício nas taxas de absenteísmo. Os efeitos em desfechos clínicos foram neutros. O efeito também foi neutro no que se refere à satisfação do paciente e à continuidade do cuidado. ${ }^{8}$ Os principais resultados desta revisão estão sistematizados na Tabela 1.

Tabela 1. Seleção dos principais resultados após implementação do Acesso Avançado, sumário dos estudos

\begin{tabular}{|c|c|c|}
\hline Desfecho & $\begin{array}{l}\text { Número de } \\
\text { estudos que } \\
\text { avaliaram }\end{array}$ & Resultado global \\
\hline $\begin{array}{l}\text { Tempo para a próxima } \\
\text { terceira-consulta-disponível }\end{array}$ & 8 & $\begin{array}{l}\text { Melhora estatisticamente significativa em } 5 \text {; qualquer melhora em todos os } 8 \text {; apenas } \\
2 \text { conseguiram garantir acesso em } 48 \mathrm{~h}\end{array}$ \\
\hline Taxa de absenteísmo & 11 & $\begin{array}{l}\text { Melhora estatisticamente significativa em } 5 \text {; mais do que } 2 \% \text { de melhora absoluta em } \\
6 \text {; qualquer melhora em } 10\end{array}$ \\
\hline Satisfação do paciente (global) & 4 & Melhora estatisticamente significativa em 1; qualquer melhoria em 2 \\
\hline $\begin{array}{l}\text { Satisfação do paciente (com o } \\
\text { agendamento) }\end{array}$ & 4 & $\begin{array}{l}\text { Nenhuma melhora estatisticamente significativa; qualquer melhora em dois; piora } \\
\text { estatisticamente significativa em } 1\end{array}$ \\
\hline Continuidade do cuidado & 9 & $\begin{array}{l}\text { Melhora estatisticamente significativa em 3; qualquer melhora em 7; piora em } 2 \\
\text { (nenhuma estatisticamente significativa) }\end{array}$ \\
\hline Utilização de serviços de saúde & 2 & $\begin{array}{l}\text { Nenhuma mudança significativa nos atendimentos em departamentos de urgência e } \\
\text { internações; } 1 \text { estudo reduziu visitas aos departamentos de urgência }\end{array}$ \\
\hline
\end{tabular}
Adaptado de Rose et al. ${ }^{8}$

A despeito da ideia que o acesso avançado privilegiaria o manejo de situações agudas, uma outra revisão avaliou o impacto desse sistema no acompanhamento de pessoas com doenças crônicas, revelando que não houve prejuízo no acompanhamento de pacientes com diabetes ou doença coronariana. ${ }^{9}$ 


\section{Acolhimento à demanda espontânea}

Um modelo de organização do processo de trabalho que vem sendo amplamente utilizado nas USF, nos diversos municípios do país, é o "acolhimento à demanda espontânea" ou "acolhimento". Esse modelo visa dar resposta às necessidades de saúde da população, ampliando o acesso das demandas urgentes para o mesmo dia, invertendo uma lógica de agendamento prévio, com grandes períodos de espera. ${ }^{10}$

Nesse modelo existe uma distinção entre "demanda espontânea" e "agenda programada". A definição do que é demanda espontânea não contempla apenas o aspecto biológico, mas depende da necessidade do usuário e da capacidade de resposta da unidade. ${ }^{10}$ Nesse modelo, também chamado de "crave-out" ou "primeira geração de acesso avançado", apenas a "demanda espontânea" terá garantida abreviação do tempo-resposta, enquanto pessoas que procuram o serviço por necessidades não urgentes continuarão tendo suas consultas adiadas para um momento futuro. ${ }^{6}$

\section{Horário estendido}

Uma forma de ampliar o acesso à APS é estender o horário de atendimento. O atendimento em horário estendido, fornecido à pessoa por seu próprio médico ou sua equipe, diminui o risco de fragmentação do cuidado, pois evita a introdução de um provedor adicional, em departamentos de emergência, que desconhece o paciente, ou não tem acesso ao seu prontuário. ${ }^{5}$ Além disso, pode ajudar a reduzir custos para o sistema de saúde como um todo, uma vez que atendimentos de emergência custam mais do que visitas a unidades de APS. ${ }^{5}$

Em nações como Reino Unido e Holanda, 95\% e 94\%, respectivamente, dos médicos de APS oferecem horários ampliados a seus usuários, enquanto nos Estados Unidos esse número é de apenas $29 \%{ }^{5}$

Não existem estudos que forneçam essa proporção no Brasil, mas muitos municípios, nas mais diversas regiões do país, vêm implantando individualmente essas modalidade de oferta por serviços de saúde: Curitiba (PR), Mogi das Cruzes (SP), Boa Vista (RR), entre outros, são exemplos. ${ }^{11-14}$ Algumas unidades de saúde da família das cidades de Boa Vista e Mogi das Cruzes têm funcionamento de 24 horas por dia. Cada cidade estabelece a organização desta oferta por meio da definição de qual população pode acessar esse serviço, dos seus dias de funcionamento e da equipe que irá atender às demandas espontâneas, se distinta ou não da equipe de saúde da família. ${ }^{11-14}$

Essa variabilidade de modelos entre serviços de APS com horário estendido também acontece em países ocidentais. ${ }^{15,16}$ Seis modelos principais, não mutuamente exclusivos, ocorrem ao redor do mundo, conforme sistematizado na Tabela 2.

\section{"Upinhas 24 horas": como funciona esse novo modelo}

Desde 2014, a prefeitura do Recife vem implantando USF com funcionamento 24 horas por dia: as "Upinhas 24 horas". Cada unidade é ocupada por três equipes de Saúde da Família (eSF), todas com equipe de saúde bucal, cada equipe com aproximadamente 3.500 usuários cadastrados. Durante a semana, segunda a sexta-feira, das 7 às 19 horas, a assistência é oferecida pelas três eSF. Nesses horários são ofertados cuidados programados e assistência à urgência apenas aos usuários cadastrados na unidade, perfazendo um total de aproximadamente 10.500 pessoas adscritas. ${ }^{17}$ 
Tabela 2. Modelos de serviços de atenção em horário estendido

\begin{tabular}{|c|c|c|c|}
\hline & Modelo Organizacional & Definição & Exemplo \\
\hline \multirow{3}{*}{$\begin{array}{l}\text { Depende de } \\
\text { adscrição de } \\
\text { clientela }\end{array}$} & $\begin{array}{l}\text { Serviço individual baseado na } \\
\text { área adscrita }\end{array}$ & $\begin{array}{l}\text { Um médico de família em sua unidade vendo seus próprios } \\
\text { pacientes em horário estendido }\end{array}$ & $\begin{array}{l}\text { Áreas rurais da } \\
\text { Austrália }\end{array}$ \\
\hline & $\begin{array}{l}\text { Serviço rotativo baseado na } \\
\text { área adscrita }\end{array}$ & $\begin{array}{l}\text { Médicos de família que trabalham numa mesma região se } \\
\text { alternam em plantões de horário estendido, cuidando da } \\
\text { população de todos os membros do grupo (cerca de } 15 \text { médicos) }\end{array}$ & $\begin{array}{l}\text { Municípios na } \\
\text { Noruega }\end{array}$ \\
\hline & $\begin{array}{l}\text { Cooperativas de médicos de } \\
\text { família }\end{array}$ & $\begin{array}{l}\text { Médicos de família trabalham em uma organização sem fins } \\
\text { lucrativos e se revezam em plantões de horário estendido para } \\
\text { a população de pacientes de todos os médicos participantes, } \\
\text { ou independente de adscrição de clientela. Estas organizações } \\
\text { são de grande porte e têm suporte de enfermeiros, gestão, } \\
\text { motoristas, etc. }\end{array}$ & $\begin{array}{l}\text { Modelo mais usado } \\
\text { na Holanda }\end{array}$ \\
\hline \multirow[t]{3}{*}{$\begin{array}{l}\text { Independente } \\
\text { de adscrição } \\
\text { de clientela }\end{array}$} & Serviços substitutos & $\begin{array}{l}\text { Empresas comerciais que empregam médicos de família para } \\
\text { prestar um serviço de horário estendido para pacientes de } \\
\text { outros médicos }\end{array}$ & $\begin{array}{l}\text { NHS Direct no } \\
\text { Reino Unido }\end{array}$ \\
\hline & Centros de APS & $\begin{array}{l}\text { Centros que os pacientes podem visitar sem um agendamento, } \\
\text { para pequenas lesões ou doenças. Tais centros operam sob a } \\
\text { supervisão de um clínico geral ou médico de família }\end{array}$ & Eslovênia \\
\hline & "Walk-in-centers" & $\begin{array}{l}\text { Centros que os pacientes podem visitar sem um agendamento, } \\
\text { com pequenas lesões ou doenças, a fim de pedir a uma } \\
\text { Enfermeira treinada informações de saúde, aconselhamento e } \\
\text { tratamento. }\end{array}$ & $\begin{array}{l}\text { Alguns modelos } \\
\text { privados na Irlanda }\end{array}$ \\
\hline \multirow[t]{2}{*}{$\begin{array}{l}\text { Não baseado } \\
\text { em APS }\end{array}$} & $\begin{array}{l}\text { Serviço de pronto-atendimento } \\
\text { hospitalar }\end{array}$ & $\begin{array}{l}\text { Pacientes de cuidados primários que utilizam o serviço de } \\
\text { emergência no horário estendido }\end{array}$ & $\begin{array}{l}\text { Modelo mais } \\
\text { comum no Brasil }\end{array}$ \\
\hline & $\begin{array}{l}\text { Serviço Telefônico de } \\
\text { aconselhamento e triagem }\end{array}$ & $\begin{array}{l}\text { Contato com um profissional com formação médica através de } \\
\text { um número fixo de telefone nacional. Esta pessoa aconselha } \\
\text { ou refere o paciente para o profissional mais adequado. }\end{array}$ & $\begin{array}{l}\text { "Call-center" } \\
\text { nacional em } \\
\text { Portugal }\end{array}$ \\
\hline
\end{tabular}

Adaptado de Huibers et al..$^{15}$ e Leibowitz et al. ${ }^{16}$

À noite, aos finais de semana e feriados, apenas o cuidado às chamadas pequenas urgências é ofertado por uma equipe de plantonistas (um médico, um enfermeiro e um técnico ou auxiliar de enfermagem), a área de abrangência é ampliada para usuários cadastrados em outras 12 equipes de outras USF do entorno, perfazendo um total de aproximadamente 50 mil pessoas. ${ }^{18}$ Atualmente, 4 "Upinhas 24 horas" estão em funcionamento, abrigando um total de 12 eSF. Ao todo, a cidade do Recife possui 122 USF ("Upinhas 24 horas" incluídas), onde atuam 268 eSF. ${ }^{18}$

O escopo de casos urgentes que podem ser atendidos na unidade é delimitado dessa forma: na entrada de cada unidade existe um cartaz expondo quais situações de urgência podem ser manejadas nestes serviços, conforme transcrição abaixo.

"Em caso de urgências, os usuários cadastrados devem procurar a Unidade para as situações de tonturas, vômitos, dores abdominais, diarreia, febre, viroses, dores de ouvido, crises leves de asma, ferimentos superficiais, resfriados, gripes, dores no peito, manchas no corpo, dores de garganta". ${ }^{17}$

As "Upinhas 24 horas" foram construídas seguindo um único projeto arquitetônico, com pequenas modificações na área externa. Nestas unidades se encontram: recepção com banheiros para usuários e sala de espera; uma sala de pré-consulta; uma sala de acolhimento; uma sala do Núcleo de Apoio ao Saúde da Família (NASF); uma sala para atividades coletivas; uma sala de vacinas; uma sala de curativos; uma sala de esterilização; uma sala de reuniões; seis consultórios, dois com sanitários e macas ginecológicas; consultório odontológico com três cadeiras de atendimento e sala de Raio X; sala de observação (com 
cinco leitos, saídas de gases, eletrocardiógrafo, desfibrilador automático, monitor/desfibrilador); sala de nebulização; sala de roupa limpa; sala de roupa suja; repouso masculino e feminino; banheiros para funcionários; sala de administração; depósito de material de limpeza (DML) e Centro de Processamento de Dados (CPD). ${ }^{11}$ Todas são informatizadas e utilizam o software Prontuário Eletrônico do Cidadão (PEC) do Sistema e-SUS Atenção Básica.

\section{Discussão}

O fator mobilizador para gestores municipais implantarem USF com funcionamento 24 horas parece ter se dado a partir da percepção de que a APS é via preferencial de acesso ao sistema de saúde, das evidências de alta utilização dos serviços de emergência por condições não urgentes, em horários nos quais as USF não estão em funcionamento, e dos resultados de estudos que descrevem experiências de organização de serviços de APS que objetivam ampliação do acesso. Faz-se necessária uma análise, à luz da literatura, da experiência em funcionamento no Recife para refletir se esse novo modelo aponta para uma ampliação de acesso ao sistema de saúde.

No caso das "Upinhas 24 horas" do Recife, observa-se que há de fato um horário estendido, para além do horário convencional de funcionamento das USF, no qual o usuário pode procurar a equipe à qual está adscrito. No entanto, o horário de sua equipe é estendido em apenas 3 horas por dia comparado aos outros serviços da APS do município, das 7 às 8 horas e das 17 às 19 horas, o que muitas vezes não contempla as pessoas que trabalham em turno convencional, tendo em vista os problemas de mobilidade da cidade.

Este modelo de horário estendido, apesar de garantir a assistência por um profissional de saúde, parece favorecer a quebra na continuidade do cuidado, pois nos finais de semana e horários noturnos, após às 19h, a chance do usuário ser atendido por seu próprio médico de família, ou por sua equipe, é pequena.

Esse problema seria diminuído se o horário das eSF fosse estendido para mais horas noturnas ou aos finais de semana. Uma revisão sistemática mostrou que, durante a semana, a maioria das pessoas usa o horário estendido entre as 18 e as 23 horas. ${ }^{19}$ Além disso, alguns usuários podem preferir os finais de semana em detrimento das horas noturnas. ${ }^{20}$

Outro ponto a se analisar é que, aos finais de semana e nos horários das 19 às 7 horas, o paciente é atendido por plantonistas, que podem ou não ser médicos da Estratégia de Saúde da Família (ESF), alguns desses plantonistas sequer trabalham com APS. Modelo esse próximo aos "serviços substitutos", descrito na Tabela 2.

Evidências apontam para que os médicos das eSF ajudem no cuidado fora do horário comercial, na forma de rodízio regional, ${ }^{21}$ o que aproximaria o serviço ao modelo de "serviço rotativo baseado na área adscrita" (Tabela 2). Dessa forma, os profissionais conheceriam melhor os problemas daquele território, e a chance de uma pessoa ser atendida por um profissional de sua equipe adscrita durante o horário estendido seria maior, favorecendo à continuidade do cuidado. ${ }^{16}$

Conforme destacado anteriormente, a ampliação do acesso não é garantida apenas com a implantação de horário estendido. ${ }^{22}$ Para que se ofereça acesso em tempo oportuno, é necessário que outras barreiras de acesso, como forma de agendamento, sejam repensadas. O modelo adotado nas unidades 24 horas é o "Acolhimento à demanda espontânea", o qual cria uma dicotomia entre "demanda espontânea" e "agenda 
programada", privilegiando a primeira. Faltam estudos que comprovem a efetividade e o benefício desse sistema. ${ }^{21}$ Privilegiar a "demanda espontânea" corrobora com outro fenômeno observado nas "Upinhas": a percepção, por parte de profissionais e usuários, de que essas unidades têm um caráter emergencista. Outras estratégias de ampliação do acesso não são estimuladas, como o acesso avançado.

Em recente revisão brasileira, o acesso avançado parece ser o modelo de agendamento mais promissor ao equilibrar demanda e capacidade controlando o tempo de espera. ${ }^{22}$ Outros modelos precisariam ser desestimulados: há "Upinhas 24 horas" onde os profissionais persistem com agendas setorizadas por grupos de risco (puericultura, gestantes, hipertensos e diabéticos); quando os esforços são concentrados nestes grupos, outras demandas são negligenciadas. AAPS de alta qualidade deve conseguir dar resposta a todas as demandas, inclusive as destes grupos. ${ }^{23}$

Numa perspectiva de ampliação de acesso à rede de APS da cidade, a implantação de serviços 24 horas precisa estar alinhada ao funcionamento das demais USF, de forma que todas elas desenvolvam ações nesse sentido. As USF do Recife, mesmo aquelas que têm uma "Upinha 24 horas" de referência, fecham suas portas por pelo menos uma hora na hora do almoço e não funcionam com horário estendido (o horário padrão é das 8 às 12 horas e das 13 às 17 horas).

Tomando como base o modelo "serviço rotativo baseado na área adscrita", a fim de que a maior parte das demandas sejam atendidas na APS, sugere-se que as USF que têm uma unidade 24 horas como referência para o horário estendido, também ampliem o acesso no horário convencional, momento em que os usuários dessas USF não podem ser atendidos na unidade 24 horas. Na Inglaterra, por exemplo, evidências mostram que a dificuldade de acesso ao médico de família no horário convencional de atendimento está associada a uma maior procura por serviços de APS de horário estendido, independentemente de outras variáveis como idade, gênero, origem étnica e condição socioeconômica. ${ }^{24}$

Em alguns países, como na Bélgica, o modelo de atendimento às urgências é centrado na APS, no qual os pacientes acessam suas unidades de saúde em horário convencional, e têm um serviço de horário estendido da APS regionalizado onde podem ser atendidos. Os departamentos de urgência hospitalar apenas complementam essa rede, com mecanismo de reembolso do valor integral do atendimento vinculado ao encaminhamento a partir de serviços de APS, ou pacientes trazidos por paramédicos em ambulâncias. ${ }^{25}$

A experiência em curso no Recife, da forma como se apresenta, parece ser baseada na concepção de uma APS que funciona como complemento para "desafogar" os serviços de atendimento às urgências já existentes e não como ordenadora do serviço de atenção às urgências. Isso pode ser evidenciado pela forma como se deu a implantação isolada de "Upinhas 24 horas" no município, sem a orientação para que as USF que fazem parte da área adscrita ampliada (que pode ser atendida à noite e finais de semana) garantam o acesso aos seus usuários no horário convencional.

Outro fator que contribui para essa percepção é o número insuficiente das "Upinhas 24 horas". Nem todas as 268 eSF possuem em seu território uma Upinha de referência onde seus usuários possam ser atendidos no horário estendido, nem foi divulgado planejamento que almeje essa cobertura. Se a proporção atual for respeitada, 50.000 usuários adscritos por Upinha no horário estendido, seriam necessárias mais 17 "Upinhas 24 horas". Além disso, não existe integração do prontuário eletrônico, uma vez que, apesar de usarem o mesmo sistema, os dados não são integrados, o que resulta em fragmentação do cuidado. 


\section{Conclusão}

Estratégias para ampliação de acesso aos cuidados de saúde parece ser um tema que interessa aos países com APS robusta. A preocupação de alguns municípios do Brasil em garantir acesso aos serviços de saúde por meio da implantação de novos modelos demonstra-se pertinente e é um passo essencial no fortalecimento de uma APS. No entanto, é preciso ter em mente que o impacto do horário estendido na ampliação do acesso pode ser minimizado se outras medidas, como mudanças no modelo de agendamento, priorizando o acesso avançado, não forem concomitantemente implantadas. Também, é preciso se pensar modelos que incentivem a continuidade do cuidado, a fim de evitar a reprodução da fragmentação que ocorre na atenção à urgência na rede de saúde já existente.

Por fim, mas não menos importante, é preciso se debruçar sobre alguns aspectos que avaliem o impacto da implantação dessas novas unidades na ampliação do acesso em sua magnitude. Questões tais quais custo-efetividade, resolutividade, perfil de problemas atendidos por outros provedores que não a equipe de saúde de origem, entre outros, ainda precisam ser respondidas. Às lentes dos modelos vigentes, apesar de apontar numa direção inicialmente acertada, a implantação das "Upinhas 24 horas" parece ainda ser uma proposta incipiente na garantia de acesso.

\section{Referências}

1. Starfield B. Atenção Primária: equilíbrio entre necessidades de saúde, serviços e tecnologia. Brasília: UNESCO, Ministério da Saúde; 2002. $726 \mathrm{p}$.

2. Gérvas J, Fernández MP. Uma Atenção Primária forte no Brasil. Relatório sobre como fortalecer os acertos e corrigir as fragilidades da Estratégia de Saúde da Família. 2011. [acesso em 2017 Set 18]. Disponível em: http://www.sbmfc.org.br/media/file/documentos/ relatoriofinal_portugues.pdf

3. Giesen P, Franssen E, Mokkink H, van den Bosch W, van Vugt A, Grol R. Patients either contacting a general practice cooperative or accident and emergency department out of hours: a comparison. Emerg Med J. 2006;23(9):731-4. DOI: http://dx.doi.org/10.1136/ emj.2005.032359

4. Fischer L, Begley C, Giardino A. The Impact of Extended Hours Primary Care on Emergency Department Use Among Medicaid/SCHIP Enrollees in Houston, TX Houston: University of Texas; [Internet] 2011. [acesso 2017 Set 19]. Disponível em: https://sph.uth.edu/content/ uploads/2011/12/Impact_of_Extended_Hours_Primary_Care.pdf

5. O'Malley AS. After-hours access to primary care practices linked with lower emergency department use and less unmet medical need Health Aff (Millwood). 2013;32(1):175-83. DOI: http://dx.doi.org/10.1377/hlthaff.2012.0494

6. Murray M, Tantau C. Same-day appointments: exploding the access paradigm. Fam Pract Manag. 2000;7(8):45-50.

7. JC Online. Upinhas ajudam na atenção básica. Recife [Internet]; 2014 Aug 26;1. [acesso 2017 Set 19]. Disponível em: http://jconline. ne10.uol.com.br/canal/cidades/saude/noticia/2014/08/26/upinhas-ajudam-na-atencao-basica-142254.php

8. Rose KD, Ross JS, Horwitz LI. Advanced access scheduling outcomes: a systematic review. Arch Intern Med. 2011;171(13):1150-9. DOI: http://dx.doi.org/10.1001/archinternmed.2011.168

9. Degani N. Impact of advanced (open) access scheduling on patients with chronic diseases: an evidence-based analysis. Ont Health Technol Assess Ser. 2013;13(7):1-48.

10. Brasil. Ministério da Saúde. Cadernos de atenção básica: Acolhimento à demanda espontânea. Brasília: Ministério da Saúde; 2011.

11. Prefeitura Municipal de Curitiba: Com 44 novas equipes do Saúde da Família, Curitiba vai ampliar horário de oito unidades de saúde - Prefeitura de Curitiba [Internet]. 2013 [Acesso 2017 abr 3]. Disponível em: http://www.curitiba.pr.gov.br/noticias/com-44-novasequipes-do-saude-da-familia-curitiba-vai-ampliar-horario-de-oito-unidades-de-saude/29004

12. Cejam: Centro de Estudos e Pesquisas “DR. João Amorim”: USF 24 Horas Jundiapeba [Internet]. [acesso 2017 Abr 3]. Disponível em: http://www.cejam.org.br/index.php?local_id=5\&uni_id=66\&fnc=unidade\&pg=servicos_saude 
13. Cejam: Centro de Estudos e Pesquisas “DR. João Amorim”: USF 24 Horas Jardim Universo [Internet]. [acesso 2017 Abr 3]. Disponível em: http://www.cejam.org.br/index.php?local_id=5\&uni_id=67\&fnc=unidade\&pg=servicos_saude

14. Unidades de Saúde oferecem horários estendidos em Boa Vista [Internet]. 2016 [acesso 2017 Abr 3]. Disponível em: http://g1.globo. com/rr/roraima/noticia/2016/06/unidades-de-saude-oferecem-horarios-estendidos-em-boa-vista.html

15. Huibers L, Giesen P, Wensing M, Grol R. Out-of-hours care in western countries: assessment of different organizational models. BMC Health Serv Res. 2009;9:105. DOI: http://dx.doi.org/10.1186/1472-6963-9-105

16. Leibowitz R, Day S, Dunt D. A systematic review of the effect of different models of after-hours primary medical care services on clinical outcome, medical workload, and patient and GP satisfaction. Fam Pract. 2003;20(3):311-7. DOI: http://dx.doi.org/10.1093/fampra/cmg313

17.Prefeitura do Recife. Nona Upinha começa a funcionar no Recife. [Internet]. [acesso 2017 Abr 3]. Disponível em: http://www2.recife. pe.gov.br/noticias/12/04/2016/nona-upinha-comeca-funcionar-no-recife

18. Prefeitura do Recife: Secretaria Municipal de Saúde. Plano Municipal De Saúde 2014-2017. Recife: Secretaria de Saúde do Recife; 2014. p. 1-84.

19. O 'Donnell CA, Foster H, Macdonald S, Burns N, Gannon M, Gannon MM, et al. Out-of-Hours Primary Medical Care: What Can Research Tell Us? Findings From a Rapid Systematic Review and Qualitative Study Address for correspondence [Internet]; 2015. [acesso 2017 Set 19]. Disponível em: https://www.rairarubiabooks.com/related-pdf-1-gp-out-of-hours-palliative-care-handover-form.html

20. O'Malley AS, Samuel D, Bond AM, Carrier E. After-hours care and its coordination with primary care in the U.S. J Gen Intern Med. 2012;27(11):1406-15.

21. Gusso GDF, Knupp D, Trindade TG, Lermen Junior N, Poli Neto P. Bases para um Novo Sanitarismo. Rev Bras Med Fam Comunidade. 2015;10(36):1-10. DOI: http://dx.doi.org/10.5712/rbmfc10(37)1255

22. Rocha SA, Bocchi SCM, Godoy MF. Acesso aos cuidados primários de saúde: revisão integrativa.Physis. 2016;26(1):87-111. DOI: http:// dx.doi.org/10.1590/S0103-73312016000100007

23. Gusso GDF, Poli Neto P. Gestão da clínica. In: Gusso G, Lopes JMC, eds. Tratado de Medicina de Família e Comunidade: Princípios, Formação e Prática. Porto Alegre: Artmed; 2012. p. 183-90.

24. Zhou Y, Abel G, Warren F, Roland M, Campbell J, Lyratzopoulos G. Do difficulties in accessing in-hours primary care predict higher use of out-of-hours GP services? Evidence from an English National Patient Survey. Emerg Med J. 2015;32(5):373-8. DOI: http://dx.doi. org/10.1136/emermed-2013-203451

25. Willems S, Peersman W, De Maeyer P, Buylaert W, De Maeseneer J, De Paepe P. The impact of neighborhood deprivation on patients' unscheduled out-of-hours healthcare seeking behavior: a cross-sectional study. BMC Fam Pract. 2013;14:136. DOI: http://dx.doi. org/10.1186/1471-2296-14-136

a Secretaria de Saúde do Recife. Recife, PE, Brasil. brunohspessoa@gmail.com (Autor correspondente); eneline.gouveia@gmail.com; brandaocorreia.isabel@gmail.com 